\title{
Clinically Actionable Cancer Somatic Variants (CACSV): A tumor interpreted dataset for analytical workflow
}

Turki M. Sobahy ( $\nabla$ tsobahy@kfshrc.edu.sa )

King Faisal Specialist Hospital \& Research Center-Jeddah (KFSHRC-J) https://orcid.org/0000-00031797-3098

\section{Donya Bahussain}

King Faisal Specialist Hospital \& Research Center-Jeddah (KFSHRC-J)

\section{Raneem Al-Harbi}

King Abdulaziz University (KAU)

\section{Method Article}

Keywords: Somatic genetic variants, AMP-ASCO-CAP recommendations, Tumor site(s), Genetic variant class, gene-tumor consensus

Posted Date: August 5th, 2021

DOl: https://doi.org/10.21203/rs.3.rs-783173/v1

License: (c) (i) This work is licensed under a Creative Commons Attribution 4.0 International License. Read Full License 


\title{
Clinically Actionable Cancer Somatic Variants (CACSV): tumor interpreted dataset for analytical workflow
}

\author{
Turki M. Sobahy ${ }^{1}$, Donya Bahussain ${ }^{1}$, Raneem Al-Harbi ${ }^{2}$ \\ ${ }^{1}$ King Faisal Specialist Hospital \& Research Center-Jeddah (KFSHRC-J), Research Center, Jeddah 21499, Kingdom of \\ Saudi Arabia \\ ${ }^{2}$ King Abdulaziz University (KAU), College of Medicine, Genetic Medicine Department, Jeddah 7393, Kingdom of Saudi \\ Arabia
}

\section{Abstract}

\section{Background:}

The recent development and enormous application of parallel sequencing technology in oncology have produced immense cell-specific genetic data. However, publicly available cell-specific genetic variants are not explained by well-established guidelines. Additionally, cell-specific variants interpretation and classification has remained a challenging task, and lacks standardization. The Association for Molecular Pathology (AMP), American Society of Clinical Oncology (ASCO), and College of American Pathologists (CAP) published the first consensus guidelines for cell-specific variants cataloging and clinical interpretation.

\section{Results:}

We developed a new method that followed the consensus recommendations, and applied our method on open source tumor-specific databases to produce clinically actionable cancer somatic variants (CACSV) dataset in integratable formats by most clinical analytical workflows. We evaluated our method with well-known classification algorithms, and found the new method to be comparable and more adhering to the recent guidelines.

\section{Conclusion:}

CACSV is a step toward cell-specific genetic variants universal interpretation, readily adaptable by most clinical laboratories pipelines and can escalate somatic variants elucidation and classification. CACSV is made free available at (https://github.com/tsobahytm/CACSV/tree/main/dataset). 


\section{Keywords}

Somatic genetic variants, AMP-ASCO-CAP recommendations, Tumor site(s), Genetic variant class, gene-tumor consensus

\section{Introduction}

Next-generation sequencing (NGS) is a recent major technological advancement in biological sciences. NGS is a high-throughput, efficient and cost-effective method in contrast to single gene-by-gene techniques and replaces assays of targeted detection by massively parallel sequencing methods. NGS technology has created a multi-dimensional data space. Indeed, sequencing has enabled the identification of new genetic determinants for multiple physiological phenotypes (1, 2). (PMID: 29592813, PMID: 31208040). It has quickly become a component of diagnostic services throughout healthcare (3). (PMID: 27066891). Genetic-based disease diagnosis, prognosis and patient management hold the promise of improved clinical outcomes and patient care $(4,5)$ (PMID: 31443496,PMID: 31926773).

Genetic information is represented as changes in the deoxyribonucleic acid (DNA) molecule known as genetic variants (ISBN: 978-0-12-404748-8). A genetic variant is an observable difference from the most commonly known nucleotide(s) at a given loci and could be either constitutional or somatic (6). (PMID: 28485371). Constitutional variants are within germ cells (hence called germline) and may pass to offspring (7, 8). (PMID: 24123875, PMID: 26551669). Germline variations are used as predictive biomarkers for some tumor diagnostics, for predisposition and for disease risk estimation (9-12). (PMID: 30113886, PMID: 29039119, PMID: 30730411, PMID: 31476665). Conversely, somatic variants occur post-fertilization and are cell specific $(7,8)$. (PMID: 24123875, PMID: 26551669). Cell-specific variants harbor many genes, including tumor drivers (13, 14) (PMID: 24037244, PMID: 29056346). Genes that are cancer drivers are behavioral: tumor intensifying (oncogenes), tumor suppressors, and other genes of duality. For instance, the NOTCH gene is an established tumor suppressor in many solid tumors such as hepatocellular carcinoma and non-melanoma skin cancer; yet NOTCH is entrenched as an oncogene in T-cell acute lymphoblastic leukemia (15). (PMID: 31900418). 
This highlights the importance of the gene's dimensions and the tumor site in identifying actionable somatic variants.

Small nucleic acid variations involving single, double or triple nucleic acid bases are more readily detectable by most NGS platforms and bioinformatic workflows than structural changes such as copy number variations (CNVs) or other chromosomal abnormalities thus making "trouble-free," accumulative, large somatic databases such as The Cancer Genome Atlas (TCGA) (https://portal.gdc.cancer.gov/ ) possible. The spatial catalogue of cell and tissue types in cancer genomic research has unfolded the complexity of carcinogenesis and tumor heterogeneity (16). (PMID: 31757986).

Cancer heterogeneity is defined as the presence of a subpopulation of cancer cells with various phenotypes and genotypes that may lead to contrastive biological behaviors within the primary tumor known as intra-tumor heterogeneity or between tumors of the same histopathological subtype which is defined as inter-tumor heterogeneity $(1,16)$. (PMID: 29592813,PMID: 31757986). The characterization of intra-tumor heterogeneity for multiple tumor samples obtained from the same patient can be referred to as spatial heterogeneity where different cancer cells exist in the same tumor site or can be temporal heterogeneity where different cancer cells can be distantly recurrent or subsequently local in the same patient (17). (PMID: 32246132)

Challenges in analyzing information in cancer genomics have been met by the development of specific tumor databases and computational tools (13, 18-20). (PMID: 24037244, PMID: 27899578, PMID: 22588877, PMID: 28912487). A wealth of genomic data has been generated and consolidated into public repositories and can stimulate ideas from big data and machine-learning researchers. The list of database examples includes the Catalogue of Somatic Mutations in Cancer (COSMIC) (https://cancer.sanger.ac.uk/cosmic ) (18). (PMID: 27899578) and cBioPortal (https://www.cbioportal.org/ ) (19). (PMID: 22588877) for cancer genomics. However, somatic-specific hubs may include impoverished or non-specific tumor diagnosis and insufficient clinically significant information (21). (PMID: 27993330). Subsequently, thoroughly reviewed cancer resources have been developed to provide more clinically actionable information. For instance, My Cancer Genome (https://www.mycancergenome.org ) (22). (PMID: 22550248) and Personalized Cancer Therapy (PCT) (https://pct.mdanderson.org) are highly curated and have direct clinical utility though they are not readily integrated into analysis pipelines or available for bulk downloads. 
Several in silico algorithms have been developed to measure the impact of small genetic variants on gene function (20, 23-26). (PMID: 28912487,PMID: 30220433,PMID: 12824425,PMID: 23315928,PMID: 30371827). Some methods perform differently in estimating the effect of germline and somatic variants such as FATHMM-MKL (20).(PMID: 28912487) which could be caused by the lack of cell-specific molecular content. Other predictive models do equally well on general and cell-specific variants like CADD, DANN, and ClinPred $(20,23)$. (PMID: 28912487, PMID: 30220433). Also, oncogenic-specific computational methods like CScape have been created to precisely evaluate the consequences of somatic variants (20). (PMID: 28912487). Other methods predict the causality of genes in cancers using different molecular phenotypes and genetic background knowledge; however, currently there is no gold standard for computational tools for classifying and interpreting cell-specific variants in tumors (16, 21). (PMID: 31757986, PMID: 27993330).

The lack of standardization in the interpretation of cancer genetic variants in clinical settings is fairly noticeable (15). (PMID: 31900418); a survey of over 44 labs revealed a discernible degree of variation in the reporting and interpretation of cancer variants (21). (PMID: 27993330). Interestingly, variants categorized by tier-systems that reflect the clinical significance and clinical utility of each variant were found to have unique proportions among the labs surveyed as $40 \%$ applied tier-of-five, $30 \%$ used tier-of-three, and $30 \%$ implemented other classification systems. Uniformity in clinically interpreting and reporting results among different laboratories is crucial for reaching a universal standard. In 2017, a multidisciplinary working group tasked with assessing the current status of NGS-based cancer testing and establishing a standardized consensus for classification, annotation, interpretation, and reporting conventions for somatic sequence variants was convened by the Association for Molecular Pathology with representation from the American College of Medical Genetics and Genomics, the American Society of Clinical Oncology, and the College of American Pathologists (21). (PMID: 27993330). They published the first joint recommendations for somatic variant interpretation in cancer known as the AMP-ASCO-CAP recommendations or guidelines.

The recommendations classify cancer somatic variants into a four-tier system based on the availability of clinical and biological background knowledge (Figure 1). Class I includes variants with strong clinical significance, class II covers variants with potential clinical significance, class III is for variants with unknown clinical significance, and class IV includes variants with significant allele frequencies in the general population. The level of evidence is 
catalogued into four groups. Group A contains genetic knowledge in oncological professional guidelines or therapeutic information for an FDA-approved drug for a particular type of cancer. Group B includes reported knowledge in well-powered studies with consensus (gene-tissue vector) by specialists. Group $\mathrm{C}$ includes knowledge about a drug investigation for a specific tumor type, an FDA-approved drug for any given tumor type, or knowledge from a few small studies with limited gene-tissue consensus. Finally, group D includes data about preclinical trials or preliminary publications with no consensus (21). (PMID: 27993330).

In our work, we adapted the AMP-ASCO-CAP recommendations into a new computational method (Figure 2). Then, we applied our classifier on publicly available somatic variants that are trouble-free by most NGS platforms and developed a clinically actionable cancer somatic variant (CACSV) database in readily integrable formats (JSON) for most analytical workflows.

\section{Methods}

\section{Level of evidence}

Identifying the source for each evidence level is important for good variant categorization. We used the National Comprehensive Cancer Network Clinical Practice Guidelines in Oncology (NCCN Guidelines $₫$ ) as our professional guidelines for clinically applicable variants. Precision Oncology Knowledge Base (OncoKB) was our source of druggable genetic variants that have approved investigative treatment or have preclinical biological evidence. The type and the level of supportive literature was collected from cBioPortal and COSMIC. Finally, the level of concurrence was measured by cataloging the knowledge of genes in the Cancer Gene Census (CGC) (https://cancer.sanger.ac.uk/census ) and Candidate Cancer Gene Database (CCGD) (http://ccgd-starrlab.oit.umn.edu/ ) against their tissue involvement (Figure 3, Supplement table 1).

\section{Text mining}

Information about actionable genetic variants in the NCCN guidelines was collected by manual extraction; only information on single genetic variants (SNVs) was extracted. There were non-specific descriptions for some variants in the NCCN guidelines; for instance, the NCCN panel suggested the use of KRAS activation variants (codon 12 and others) as 
therapeutic and prognostic biomarkers for non-small-cell lung carcinoma (NSCLC) patients. Consequently, only kinase domain KRAS variants with high confidence predictive scores (CScape) and predicted tumor drivers (intOgen) were selected. Other examples are hyper-mutated genes like the BRCAs (1/2) variants in ovarian cancers. The NCCN panel discussed the use of BRCA changes (germline or somatic) to inform maintenance therapy and TP53 variants in acute myeloid leukemia (AML) patients. The AML panel suggested the use of such variants as prognostic biomarkers. Genes harboring many mixed (germline and somatic) variants require careful curation. We used single-gene expert-reviewed databases (BRCA Exchange (BRCAEx), International Agency for Research on Cancer (IARC)) to expand upon those variants. Only BRCAEx (https://brcaexchange.org/ ) summary view pathogenic variants were selected. IARC-TP53 (https://p53.iarc.fr/ ) somatic variants were selected only if reported from the hematopoietic system as the primary site. In addition, MET exon 14 skipping variants in NSCLC guidelines were recommended to be used as therapeutic biomarkers for Crizotinib (as category $2 \mathrm{~A}$ ). These types of variants require specific laboratory validation. We collected only the skipping variants that had undergone experimental validation $(27,28)$. (PMID: 25971938,PMID: 25971939). As a result, an expanded file for the unspecified genetic variants in the NCCN guidelines was developed (Supplement table 2)

\section{Data acquisition and integration}

OncoKB (https://www.oncokb.org/ ) was used as an information source about therapeutic options (Nov, 2020). We encountered the same challenge for some genetic variants that have generic descriptions. For example, EGFR gene exon 19 deletion and NRAS oncogenic mutations were listed as actionable variants. Only variants that mentioned the same consequence and were predicted to be deleterious by tumor-specific methods CScape (http://cscape.biocompute.org.uk/ ) and IntOgen (https://www.intogen.org/search ) were selected (Table 1, Supplement table 3) (Nov, 2020).

Then, cancer genetic variant hubs were downloaded. The oncotree was used as a universal tissue spatial tree for different tumors. For each database, tumor sites were mapped to the nearest histopathology and cell type on the universal tree model (http://oncotree.mskcc.org/ ) (Table 2). We focused on seven major cancer sites in our work: bowel, breast, brain/central nervous system (CNS), esophagus/stomach, skin, lung and pancreas. The latest COSMIC version dataset was obtained in July 2020, intOgen's latest version, and the bulk dataset of cBioPortal was downloaded in August 2020. In the cBioPortal dataset, genetic variants with no 
mentioned or specified tumor sites or that had low-quality samples with respect to tumor cellularity were deemed "problematic" and excluded.

PanCan studies were considered to be well-powered research while those remaining were deemed small studies. A variant supported by more than 5 single studies was considered to be from multiple findings while those with fewer than 5 were classified as a few reports (Figure 2). Consensus was defined based on a gene's candidacy as a tumor driver given tissue type using expert-curated resources. Census scores were developed to reflect the degree of consensus per tumor site given a single gene. The score depends on the consistency of reviewed knowledge between a gene and a distinct cancer tissue. For this purpose, we used the Cancer Gene Census (CGC), and Candidate Cancer Gene Database (CCGD). A list of all available genes from both sources was collected. Next, all tissue types were aligned with the universal oncotree model (Table 2), and then multiple gene-tissue vectors were generated for each gene. CGC-based vectors were given higher ranking (consensus score =1) and CCGD vectors were given consensus scores of 2 , otherwise vectors were denoted with a value of zero. (Supplement Table 1).

\section{Results}

\section{Testing dataset}

We piled up all somatic variants on the above mentioned databases into a single collective list. Then, all variants that intersected with the gnomAD (https://gnomad.broadinstitute.org/ ) database were removed, leaving about 2,952,167 somatic variants. Finally, we simulated the list with all 11 selected tumor sites, producing 10 specific tumor-site datasets (Figure 4)

\section{Classification}

Every simulated dataset was interpreted and catalogued by the classifier we developed. Since germline-based filtration was done, our classifier ranked only the first three tiers of the AMP-ASCO-CAP guidelines (Table 3). The tumor tissue with the most potential actionable variants (tiers I \& II) was the CNS with 413 variants while 4 tumor sites-anal, esophageal, gastric and pancreas-showed no somatic variants on tier I and had the lowest potentially actionable variants (338). All of the class II variants in the tumors had level D evidence of 
preclinical trials or biological attestation in solid tumors. Most of the tier I variants were ranked that way because they are mentioned in the NCCN guidelines in the generic descriptions and are identified as tumor drivers (oncogenic) by intOgen and CScape (Supplement Table 2).

\section{Comparison with other tools.}

Publicly available software that adapts and follows the AMP-ASCO-CAP recommendations (29). (PMID: 31443733) is limited. Two methods were selected for comparative analysis: variant interpretation for cancer (VIC) (https://github.com/HGLab/VIC ) (29). (PMID: 31443733), and the cancer genome interpreter (CGI) (https://www.cancergenomeinterpreter.org/home ) (1). (PMID: 29592813). VIC, which was developed to classify somatic variants according to the recommended 4-tier system, was published in Aug 2019. The CGI also ranks somatic variants by a tier-of-4 metric, however, it doesn't strictly follow the recommendations (Table 4). CGI provides a hierarchical structure for the tumor sites, while VIC lays out a list of tumor sites to the user $(1,29)$. (PMID:

29592813,PMID: 31443733).

\section{Round One}

Only variants identified as tier I or II by our method were collected for the first comparative analysis (Table 5). The anal dataset was excluded from the comparison because of the absence of its equivalent in VIC and CGI. There were noticeable discrepancies between our method and the others in all datasets. For example, out of the 431 potentially actionable variants in the CNS dataset, VIC classified only one variant as tier II, 254 as variants with uncertain significance, and 158 as unable to classify. CGI categorized 390 variants: 306 predicted as drivers, 7 predicted as messengers, and 77 as known-in. In the breast dataset, our classifier identified 391 variants as potentially actionable; VIC catalogued 44 as potentially actionable (all class II), 198 with uncertain significance, and didn't catalogue 149 and CGI categorized 367, 294 as drivers, and 73 as known-in.

\section{Round Two}

The second comparative analysis with our method was on variants with uncertain significance. Random selection was done on 100 variants in each of 100 iterations returning about 10,000 variants per site-specific dataset because of the large size of this class of variants. VIC was applied on all randomized datasets and on average ranked only five variants as potentially significant (class II) (Table 6). In the breast dataset, three variants were categorized as potentially clinically significant. Two variants (CBL:NM_005188:exon9:c.G1366A:p.D456N 
and RAD21:NM_006265:exon3:c.T244A:p.F82I) are known to be on slightly hyper-mutated genes (30). (PMID: 28890946) but have no actionable variants in breast cancers and are associated with other tumor sites on CGC (Table 7). The third variant (SETBP1:NM_015559:exon6:c.A4310G:p.K1437R) gene has no supportive evidence for actionable variants in breast cancers (30). (PMID: 28890946) and is associated with myeloid malignancies on CGC. Additionally, VIC catalogued 11 variants with potential clinical significance in the colon randomized dataset. Three out of the 11 variants harbor the same

gene: BAP1: BAP1:NM_004656:exon9:c.G673C:p.D225H, BAP1:NM_004656:exon9:c.G680C:p.R227P, and BAP1:NM_004656:exon5:c.G295A:p.V99M. BAP1 is slightly hyper-mutated in colorectal cancer with no actionable variants in colon/colorectal cancers (30). (PMID: 28890946) and has no consensus with colon cancer on CGC. The AMP-ASCO-CAP recommendations require consensus on mutated genes and tumor tissue by experts in the field. VIC also categorized one SMAD4 variant (SMAD4:NM_005359:exon10:c.G1151A:p.G384D) as potentially significant. SMAD4 is known to be hyper-mutated in colorectal cancer, has no known actionable variants in colon/colorectal cancers (30). (PMID: 28890946), and has consensus with colorectal cancer on CGC. In addition, this variant is predicted as deleterious with high confidence by Cscape; however, the AMP-ASCO-CAP guidelines require a variant catalogued as tier $I$ to be reported in well-powered studies and multiple or a few reports with the same tumor site. The SMAD4 variant is found only on PAN and in single studies for esophageal carcinoma (19). (PMID: 22588877) (Table 7).

\section{Discussion}

The application of parallel sequencing in oncology for research and diagnostics has created immense databases (31, 32). (PMID: 29165669,PMID: 32461654). The availability of multiple, expert-reviewed sources for oncogenetics has deepened our understanding of tumorigenesis and expanded the known tumor molecular networks. In addition, the development of many genetic variant predictive models and classifiers has helped in filtering, classifying, and interpreting cancer genetic variants in clinical settings. Nonetheless, observable inconsistencies in routine workflows has created a demand for a clinical standard (21). (PMID: 27993330). The AMP-ASCO-CAP recommendations provide a ubiquitous approach to cataloguing cancer genetic variants. The guidelines suggest extrapolating the ACMG/AMP recommendations (33). (PMID: 25741868) for interpreting constitutional variations and propose a new algorithm for 
tumor-specific variants. In this study, we want to adapt and apply the AMP-ASCO-CAP recommendations on publicly available somatic variants and provide multiple tumor site-specific (simulated) datasets with potentially actionable clinical somatic variants.

In our attempt to follow the recommended guidelines, we needed to define some of the requirements for achieving amenable adherence and reducing ambiguity. The AMP-ASCO-CAP recommendations suggested the use of medical guidelines as a source of high confidence with clinical utility; however, multiple oncological professional guidelines are used in clinical practice. The list includes: American Society of Clinical Oncology (ASCO), European Society for Medical Oncology (ESMO), and National Comprehensive Cancer Network Clinical Practice Guidelines in Oncology (NCCN Guidelines ${ }^{\circledR}$ ). Understandably, subtle yet detectable variations in regional and institutional guidelines exist, in particular in those about genetic screening and treatment options (34). (PMID: 30962720). For example, eight guidelines suggested treatments for breast cancer (BC) patients with BRCA variants or with high familial risk for developing BC. Three guidelines suggested the use of platinum therapy as neoadjuvant therapy for BRCA-mutated metastatic $\mathrm{BC}$ for women under the age of 40 while the NCCN guidelines recommended the use of the PARP inhibitor olaparib for treatment of BRCA-mutated HER2-negative BC (34). (PMID: 30962720). Ideally, congruent clinical protocols would provide better and standardized healthcare delivery. Hence, we chose a single oncological professional guideline for our current work.

We noticed a finite number of significant variants on the datasets tested. This was not unexpected given how stringent the AMP-ASCO-CAP recommendations are. For example, somatic variants need to be discussed in oncologists' professional guidelines or supported by a good amount of consistency for a gene in a specific tissue to be ranked as clinically actionable; the professional guidelines contain a limited number of validated actionable somatic variants. However, with the current progress and data accumulation from oncogenomic research projects, we can identify novel candidate variants that are now in gray areas.

The discrepancy between our classifier and other methods was also anticipated. The CGI ranks somatic variants based on level of evidence from manually curated genomic databases, including ClinVar (31).(PMID: 29165669). In addition, tumor-driver genes were predicted using the analysis of large cancer cohorts such as The Cancer Genome Atlas and the International Cancer Genome Consortium (https://icgc.org/ ) (35).(PMID: 32025007) and then verified by the availability of experimental or computational evidence. While ClinVar provides clinical and experimental evidence for germline genetic variants, it lacks major contributions of somatic alterations. The AMP-ASCO-CAP guidelines suggested the use of ClinVar for tumor 
germline variants in the current state (21). (PMID: 27993330). The guidelines also do not count in silico analysis for any cohort size or the score of predictive models as sufficient evidence for clinical action (21). (PMID: 27993330). VIC uses prediction scores from multiple methods and uses consistency among at least four in their algorithm criteria. We, on the other hand, use only somatic variant predictive models (as they are shown to have better false positive rates (20). (PMID: 28912487)) and only to verify oncogenic variants in genes that are reported in the professional guidelines. We suggest caution in interpreting these variants as they may require additional experimental verification. In addition, VIC provides a more dynamic option for variant interpretation through manual inputs by users which would change variant classifications from the "default" settings.

We acknowledge some limitations in the CACSV database. First, there was no consideration for tumor pathway involvement. Cancer molecular networks are complex and frequently evolving; a good implementation of tumor-specific networks would allow for better variant classification. In addition, consensus guidelines recommend fine mapping to the nearest cell type: the analytical principal in the interpretation of variants with unknown significance. We plan to address these shortcomings in future updates of the CACSV. Also, oncologists' professional guidelines provide a range of therapeutic biomarkers including gene expressions, fusions and translocations while our approach covers only small DNA genetic variants as some of the other biomarkers are not easily detectable by current parallel sequencing methods and are screened by other molecular assays. Prospective CACSV releases will cover other complex biomarkers; the availability of tumor-specific, FDA-approved treatments or investigative therapies is a key criteria in the AMP-ASCO-CAP guidelines. In our current work, OncoKB was used for existing information about active or approved treatments. Adapting consensus guidelines should provide a global knowledgebase of all available treatments. We will include other international resources in the future, e.g. the University Hospital Medical Information Network (UMIN) (https://www.umin.ac.jp/english/ ) (36). (PMID: 22504688). We are also working on developing a user graphical interface to the CACSV to reach a wider range of users. Finally, expert manual review is the current gold standard for classifying and interpreting actionable somatic variants. 


\section{Conclusion}

We developed a simulated database (CACSV) for multiple tumors that catalogued publicly available cancer single nucleotide variants according to our new adaptation of AMP-ASCO-CAP recommendations. The fully classified dataset is available in integrable formats (JSON, CSV) for most clinical bioinformatics interpretation pipelines in diagnostic or oncological research settings and will help reduce post variants calling-processing and filtration. CACSV is readily available in standard serialized formats at https://github.com/tsobahytm/CACSV/tree/main/dataset.

\section{Abbreviations}

AMP: The Association for Molecular Pathology

ASCO: American Society of Clinical Oncology

CAP: College of American Pathologists

CACSV: Clinically Actionable Cancer Somatic Variants

NGS: Next-Generation Sequencing

CNVs: Copy Number Variations

TCGA: The Cancer Genome Atlas

COSMIC: Catalogue of Somatic Mutations in Cancer

PCT: Personalized Cancer Therapy

NCCN: National Comprehensive Cancer Network Clinical Practice Guidelines in Oncology

OncoKB: Precision Oncology Knowledge Base

CGC: Cancer Gene Census

CCGD: Candidate Cancer Gene Database 
SNVs: Single Genetic Variants

AML: Acute Myeloid Leukemia

IARC: International Agency for Research on Cancer

BRCAEx: BRCA Exchange

CNS: Central Nervous System

VIC: Variant Interpretation for Cancer

CGI: Cancer Genome Interpreter

ACMG: American College of Medical Genetics

UMIN: University Hospital Medical Information Network

\section{Declaration}

\section{Ethics approval and consent to participate}

Not applicable

\section{Consent for publication}

Not applicable

Availability of data and materials

The final datasets are available at GitHub (https://github.com/tsobahytm/CACSV/tree/main/dataset). All Python programs used to generate the final output are also available (https://github.com/tsobahytm/CACSV/tree/main/scripts ). The README file contains more details about each program and dataset. All somatic variants databases or tools used in the analysis and comparative testing can be found via the following URLs.

COSMIC: https://cancer.sanger.ac.uk/cosmic

cBioPortal: https://www.cbioportal.org/ 
CGC: $\underline{\text { https://cancer.sanger.ac.uk/census }}$

CCGD: http://ccgd-starrlab.oit.umn.edu/

BRCAEx: https://brcaexchange.org/

IARC-TP53: https://p53.iarc.fr/

OncoKB: https://www.oncokb.org/

CScape: http://cscape.biocompute.org.uk/

IntOgen: https://www.intogen.org/search

gnomAD: https://gnomad.broadinstitute.org/

VIC: https://github.com/HGLab/VIC

CGI: https://www.cancergenomeinterpreter.org/home

\section{Competing interests}

The authors declare that they have no competing interests.

\section{Funding}

T.M.S, D.B and R.H supported by King Faisal Specialist Hospital \& Research Center-Jeddah (KFSHRC-J)

\section{Authors' contributions}

T.M.S conceptualized the research idea, and strategized the work. R.H and D.B extracted and verified relevant text manually. T.M.S verified the extracted information. T.M.S developed all the used Python scripts and performed the comparative testing. R.H verified the testing outcomes. All figures and tables generated by T.M.S. R.H, D.B \& T.M.S contributed to the writing of the manuscript. All of the authors have read and approved the final manuscript.

\section{Acknowledgements}


For computer time, this research used the resources of the Supercomputing Laboratory at King Abdullah University of Science \& Technology (KAUST) in Thuwal, Saudi Arabia.

The authors would like to thank the following: Samar A. Zailaie for reviewing the manuscript, Emily L. Heaphy for manuscript English review, Dalia Anbari for references formatting, Saja Basha and Salouf Al-Madani for their help in results tables design.

\section{Authors' information (optional)}

The authors Donya Bahussain and Raneem Al-Harbi contributed equally to this work.

\section{References}

1. Tamborero D, Rubio-Perez C, Deu-Pons J, Schroeder MP, Vivancos A, Rovira A, et al. Cancer Genome Interpreter annotates the biological and clinical relevance of tumor alterations. Genome medicine. 2018;10(1):25.

2. Coccaro N, Anelli L, Zagaria A, Specchia G, Albano F. Next-Generation Sequencing in Acute Lymphoblastic Leukemia. Int J Mol Sci [Internet]. 2019 2019/06//; 20(12). Available from: http://europepmc.org/abstract/MED/31208040 https://doi.org/10.3390/ijms20122929 https://europepmc.org/articles/PMC6627957 https://europepmc.org/articles/PMC6627957?pdf=render.

3. Rabbani B, Nakaoka H, Akhondzadeh S, Tekin M, Mahdieh N. Next generation sequencing: implications in personalized medicine and pharmacogenomics. Molecular bioSystems. 2016;12(6):1818-30.

4. Fernandes MGO, Jacob M, Martins N, Moura CS, Guimarães S, Reis JP, et al. Targeted Gene Next-Generation Sequencing Panel in Patients with Advanced Lung Adenocarcinoma: Paving the Way for Clinical Implementation. Cancers (Basel). 2019;11(9).

5. Liu J, Liu Y. Molecular diagnostic characteristics based on the next generation sequencing in lung cancer and its relationship with the expression of PD-L1. Pathology, research and practice. 2020;216(2):152797.

6. Milholland B, Dong X, Zhang L, Hao X, Suh Y, Vijg J. Differences between germline and somatic mutation rates in humans and mice. Nature communications. 2017;8:15183.

7. Li R, Montpetit A, Rousseau M, Wu SY, Greenwood CM, Spector TD, et al. Somatic point mutations occurring early in development: a monozygotic twin study. J Med Genet. 2014;51(1):28-34.

8. Alexandrov LB, Jones PH, Wedge DC, Sale JE, Campbell PJ, Nik-Zainal S, et al. Clock-like mutational processes in human somatic cells. Nat Genet. 2015;47(12):1402-7.

9. Panou V, Gadiraju M, Wolin A, Weipert CM, Skarda E, Husain AN, et al. Frequency of Germline Mutations in Cancer Susceptibility Genes in Malignant Mesothelioma. Journal of clinical oncology : official journal of the American Society of Clinical Oncology. 2018;36(28):2863-71.

10. Schon K, Tischkowitz M. Clinical implications of germline mutations in breast cancer: TP53. Breast cancer research and treatment. 2018;167(2):417-23. 
11. Das S, Salami SS, Spratt DE, Kaffenberger SD, Jacobs MF, Morgan TM. Bringing Prostate Cancer Germline Genetics into Clinical Practice. The Journal of urology. 2019;202(2):223-30.

12. Xu K, Shi Y, Wang X, Chen Y, Tang L, Guan X. A novel BRCA1 germline mutation promotes triple-negative breast cancer cells progression and enhances sensitivity to DNA damage agents. Cancer genetics. 2019;239:26-32.

13. Gonzalez-Perez A, Perez-Llamas C, Deu-Pons J, Tamborero D, Schroeder MP, Jene-Sanz A, et al. IntOGen-mutations identifies cancer drivers across tumor types. Nature methods. 2013;10(11):1081-2.

14. Martincorena I, Raine KM, Gerstung M, Dawson KJ, Haase K, Van Loo P, et al. Universal Patterns of Selection in Cancer and Somatic Tissues. Cell. 2017;171(5):1029-41.e21.

15. Colaprico A, Olsen C, Bailey MH, Odom GJ, Terkelsen T, Silva TC, et al. Interpreting pathways to discover cancer driver genes with Moonlight. Nature communications. 2020;11(1):69.

16. Althubaiti S, Karwath A, Dallol A, Noor A, Alkhayyat SS, Alwassia R, et al. Ontology-based prediction of cancer driver genes. Sci Rep. 2019;9(1):17405.

17. Wagner AH, Walsh B, Mayfield G, Tamborero D, Sonkin D, Krysiak K, et al. A harmonized meta-knowledgebase of clinical interpretations of somatic genomic variants in cancer. Nat Genet. 2020;52(4):448-57.

18. Forbes SA, Beare D, Boutselakis H, Bamford S, Bindal N, Tate J, et al. COSMIC: somatic cancer genetics at high-resolution. Nucleic Acids Res. 2017;45(D1):D777-d83.

19. Cerami E, Gao J, Dogrusoz U, Gross BE, Sumer SO, Aksoy BA, et al. The cBio cancer genomics portal: an open platform for exploring multidimensional cancer genomics data. Cancer discovery. 2012;2(5):401-4.

20. Rogers MF, Shihab HA, Gaunt TR, Campbell C. CScape: a tool for predicting oncogenic single-point mutations in the cancer genome. Sci Rep. 2017;7(1):11597.

21. Li MM, Datto M, Duncavage EJ, Kulkarni S, Lindeman NI, Roy S, et al. Standards and Guidelines for the Interpretation and Reporting of Sequence Variants in Cancer: A Joint Consensus Recommendation of the Association for Molecular Pathology, American Society of Clinical Oncology, and College of American Pathologists. The Journal of molecular diagnostics : JMD. 2017;19(1):4-23.

22. Pao W. New approaches to targeted therapy in lung cancer. Proceedings of the American Thoracic Society. 2012;9(2):72-3.

23. Alirezaie N, Kernohan KD, Hartley T, Majewski J, Hocking TD. ClinPred: Prediction Tool to Identify Disease-Relevant Nonsynonymous Single-Nucleotide Variants. Am J Hum Genet. 2018;103(4):474-83.

24. $\mathrm{Ng}$ PC, Henikoff S. SIFT: Predicting amino acid changes that affect protein function. Nucleic Acids Res. 2003;31(13):3812-4.

25. Adzhubei I, Jordan DM, Sunyaev SR. Predicting functional effect of human missense mutations using PolyPhen-2. Current protocols in human genetics. 2013;Chapter 7:Unit7.20.

26. Rentzsch P, Witten D, Cooper GM, Shendure J, Kircher M. CADD: predicting the deleteriousness of variants throughout the human genome. Nucleic Acids Res. 2019;47(D1):D886-d94.

27. Frampton GM, Ali SM, Rosenzweig M, Chmielecki J, Lu X, Bauer TM, et al. Activation of MET via diverse exon 14 splicing alterations occurs in multiple tumor types and confers clinical sensitivity to MET inhibitors. Cancer discovery. 2015;5(8):850-9.

28. Paik PK, Drilon A, Fan PD, Yu H, Rekhtman N, Ginsberg MS, et al. Response to MET inhibitors in patients with stage IV lung adenocarcinomas harboring MET mutations causing exon 14 skipping. Cancer discovery. 2015;5(8):842-9. 
29. He MM, Li Q, Yan M, Cao H, Hu Y, He KY, et al. Variant Interpretation for Cancer (VIC): a computational tool for assessing clinical impacts of somatic variants. Genome medicine. 2019;11(1):53.

30. Chakravarty D, Gao J, Phillips SM, Kundra R, Zhang H, Wang J, et al. OncoKB: A Precision Oncology Knowledge Base. JCO precision oncology. 2017;2017.

31. Landrum MJ, Lee JM, Benson M, Brown GR, Chao C, Chitipiralla S, et al. ClinVar: improving access to variant interpretations and supporting evidence. Nucleic Acids Res. 2018;46(D1):D1062-d7.

32. Karczewski KJ, Francioli LC, Tiao G, Cummings BB, Alföldi J, Wang Q, et al. The mutational constraint spectrum quantified from variation in 141,456 humans. Nature. 2020;581(7809):434-43.

33. Richards S, Aziz N, Bale S, Bick D, Das S, Gastier-Foster J, et al. Standards and guidelines for the interpretation of sequence variants: a joint consensus recommendation of the American College of Medical Genetics and Genomics and the Association for Molecular Pathology. Genetics in medicine : official journal of the American College of Medical Genetics. 2015;17(5):405-24.

34. Forbes C, Fayter D, de Kock S, Quek RG. A systematic review of international guidelines and recommendations for the genetic screening, diagnosis, genetic counseling, and treatment of BRCA-mutated breast cancer. Cancer Manag Res. 2019;11:2321-37.

35. Pan-cancer analysis of whole genomes. Nature. 2020;578(7793):82-93.

36. Sone M, Arai Y, Kiuchi T, Ishikawa H, Aoki N, Inaba Y, et al. [Shared web-based data center for multi-institutional clinical trials: evaluation of UMIN-INDICE (university hospital medical information network-internet data and information center for medical research)in clinical trials of JIVROSG (Japan interventional radiology in oncology study group)]. Gan To Kagaku Ryoho. 2012;39(4):619-23. 


\section{Figures}

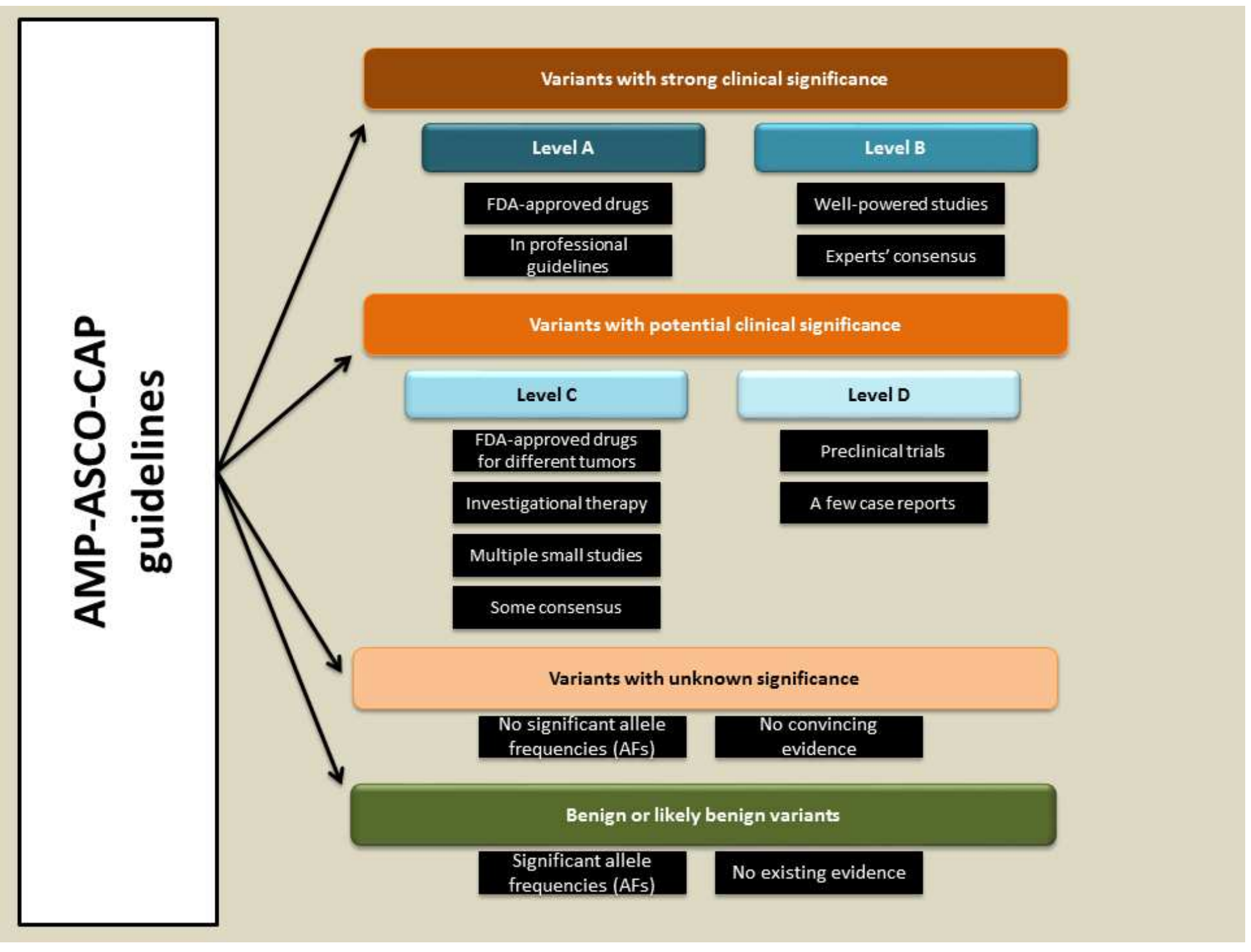

Figure 1

The current AMP-ASCO-CAP consensus guidelines for genetic variants interpretation in tumors. 


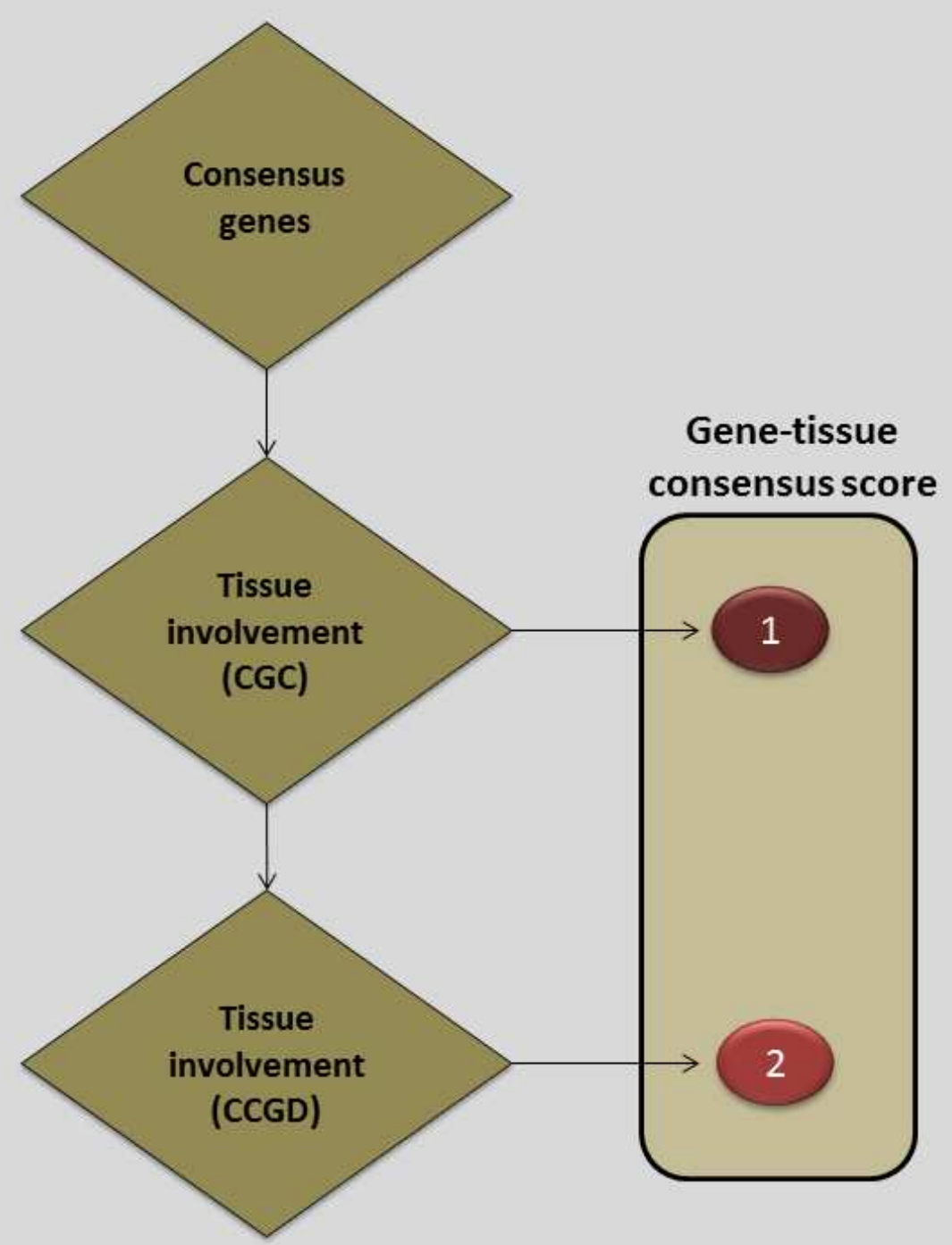

Figure 2

The adaptive algorithm for the AMP-ASCO-CAP recommendations. 


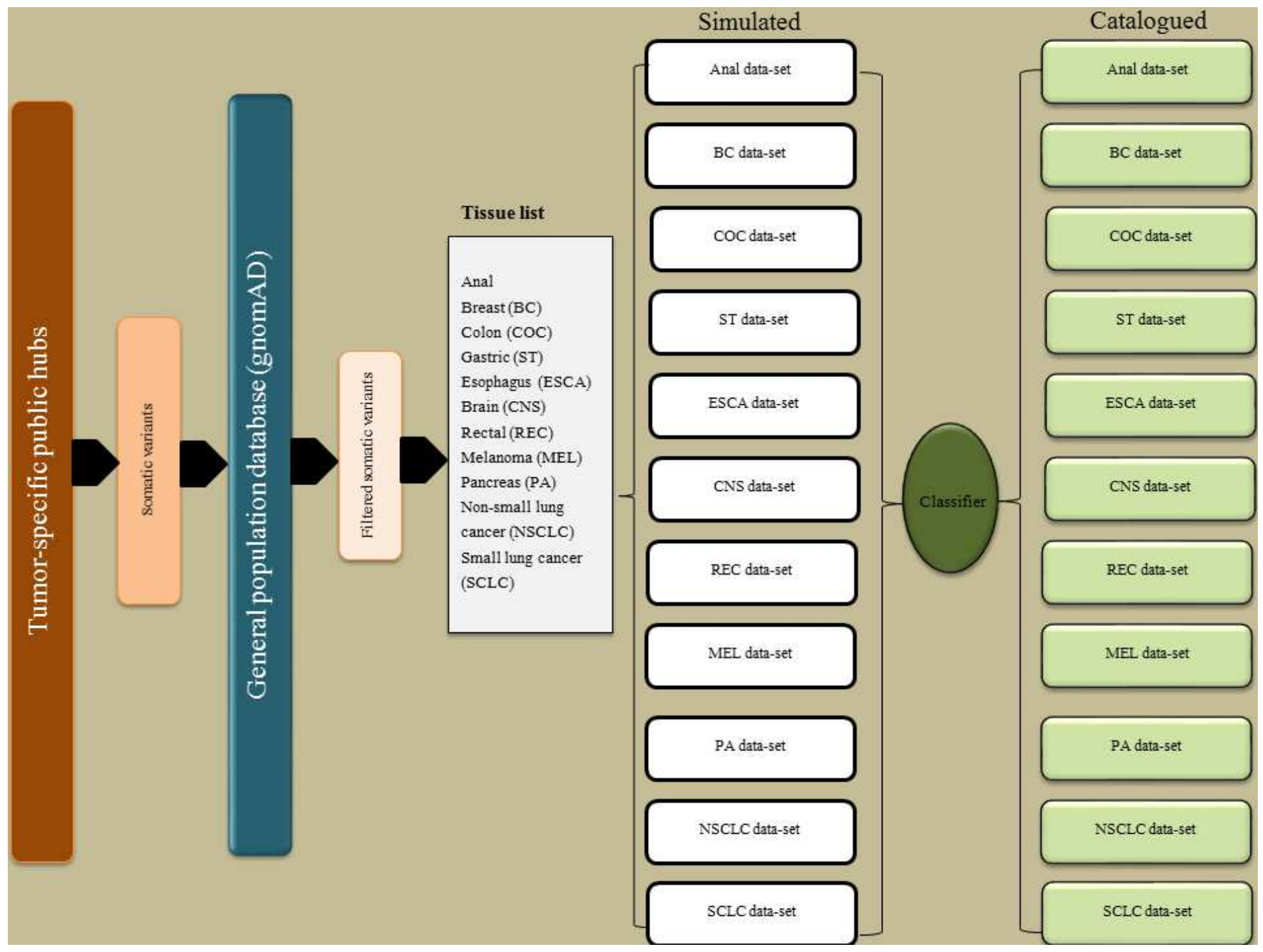

Figure 3

The developed algorithm for measuring cancer genes consensus in different cells of origins. CGC tiers I and II are equally ranked. 


\section{Strong clinical evidence}

FDA-approved

(OncoKB level 1)

Professional guidelines $(\mathrm{NCCN})$
Pan Study in the same tumor site (cBioPortal)

CGC tier I or II for the same tumor

\section{Potential clinical evidence}
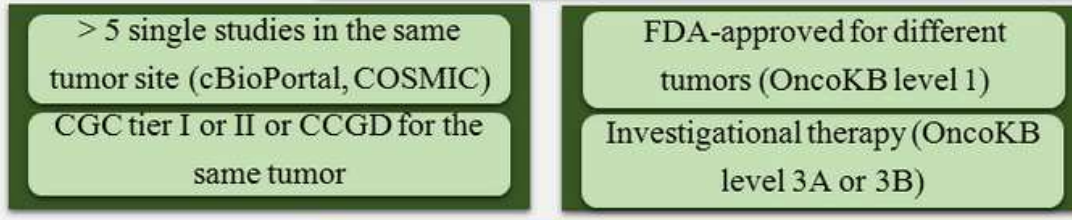

$<5$ single studies in the same tumor site (cBioPortal, COSMIC)

Preclinical evidence for the same, or all solid tumors (OncoKB level 4)

\section{Uncertain evidence}

$<5$ single studies in other tumors

(cBioPortal, COSMIC)

\section{Figure 4}

Summary of the testing data acquisition and processing.

\section{Supplementary Files}

This is a list of supplementary files associated with this preprint. Click to download.

- Tables1.docx

- Tables2.docx

- Tables3.docx

- Tables4.docx

- Tables5.docx

- Tables6.docx

- Tables7.docx

- STable1.xlsx

- STable2.xlsx

- STable3.xlsx 